\title{
Describing Quantum Circuits with Systolic Arrays
}

\author{
Aasavari Bhave and Eurípides Montagne \\ School of Electrical Engineering and Computer Science \\ University of Central Florida, Orlando, FL \\ E-mail:\{aasavari, eurip\}@cs.ucf.edu
}

\author{
Edgar Granados \\ 1677 Calabasa Dr., \\ Lincoln, CA 95648 \\ E-mail\{ergv\}@aol.com
}

\begin{abstract}
In the simulation of quantum circuits the matrices and vectors used to represent unitary operations and qubit states grow exponentially as the number of qubits increase. For instance, the evolution of an n-qubit quantum system in an initial superposition state is described by a $2^{n} \times 2^{n}$ unitary matrix. However, the evolution of an n-qubit quantum system can be described as well as a composition of single-qubit and controlled-not unitary operations which are equivalent to the $2^{n} \times 2^{n}$ unitary matrix. A strategy is suggested for the mapping of onequibit and two-qubit gates onto a three PE systolic array, and then we show how the interconnection of those systolic arrays can be used to implement or describe quantum circuits. As a case study we present the description of the teleportation algorithm.
\end{abstract}

\section{Introduction}

Since the discovery of quantum algorithms able to solve problems considered untractable on classic computers [5], the field of quantum computation and quantum information has become an integrated part of computer science due to the profound changes that quantum mechanics is bringing into the theory of computation[1]. If well the construction of a practical quantum computer has not been achieved, the search for new quantum algorithms and a new pedagogy to teach the fundamentals of quantum mechanics to computer scientists are indeed hot research areas nowadays; hence, the simulation of quantum algorithms has become a growing field of research.

The state-vector model representing the unitary transformations of the quantum state as it pass through the gates and circuits has been considered the best candidate for emulation of quantum circuits on a classical computer [6], [10]. Different approaches have been taken to simulate quantum gates and circuits ranging from Programming Languages [11], Hardware Description Language [7], Field Programmable Gate Array[8], and Decision Diagrams [9] among others simulation techniques. This paper presents the mapping of unitary matrices onto a linear systolic array[2]. We show how to apply two Hadamard operations in parallel, using a single linear systolic array. Furthermore, the same systolic array is used to implement the reversible CNOT gate. We are limiting our study of quantum registers to just two qubits because the evolution of an n-qubit quantum system can be described as well as a composition of single-qubit and controlled-not unitary operations which are equivalent to the $2^{n} \times 2^{n}$ quantum system.

The remainder of this paper is organized as follows: Section 2 provides the necessary background on quantum systems and tensor operations. Then in Section 3, we show how systolic arrays can be used to describe quantum gates operations. In Section 4, we describe a teleportation circuit using systolic arrays. Final remarks and ongoing work is presented in Section 5.

\section{Quantum Circuit Model}

A qubit is a two level quantum system which can be considered as an abstract entity or mathematical object that resides in a two-dimensional Hilbert space. This abstract object, the qubit, can be imagined as a unit vector that moves among the quantum states of a quantum system. Hence, we could say that a qubit describes a state in a two-dimensional quantum system. We can simulate a classical bit in a quantum system using a pair of mutually orthonormal state vectors as follows:

$$
|0\rangle=\left[\begin{array}{l}
1 \\
0
\end{array}\right] \text { and }|1\rangle=\left[\begin{array}{l}
0 \\
1
\end{array}\right]
$$

where the symbols $|0\rangle$ and $|1\rangle$, known as ket zero and ket one according to Dirac's notation, are labels attached to each quantum state. The states $|0\rangle$ and $|1\rangle$ form a computational basis and any other state the qubit can be in is known as a superposition and can be expressed as a linear combination of the basis states:

$$
|\psi\rangle=\alpha|0\rangle+\beta|1\rangle,
$$

where $\alpha, \beta \in \mathbb{C}$ are weighting factors also known as probability amplitudes and $|\alpha|^{2}+|\beta|^{2}=1$.

To move a qubit from one state to another we must use unitary transformations which are represented by $2 \times 2$ matrices and we will refer to them as unitary operations or gates. For instance, Pauli matrices (X, Z) along with Identity (I), are gates that can be used to act on one-qubit to describe the evolution of a quantum system. In their matrix form they are represented as:

$$
X=\left[\begin{array}{ll}
0 & 1 \\
1 & 0
\end{array}\right] Z=\left[\begin{array}{rr}
1 & 0 \\
0 & -1
\end{array}\right] I=\left[\begin{array}{ll}
1 & 0 \\
0 & 1
\end{array}\right]
$$


The unitary operation Pauli-X flips $|0\rangle$ into $|1\rangle$ and vise versa:

$$
\begin{aligned}
& X|0\rangle=\left[\begin{array}{ll}
0 & 1 \\
1 & 0
\end{array}\right]\left[\begin{array}{l}
1 \\
0
\end{array}\right]=\left[\begin{array}{l}
0 \\
1
\end{array}\right]=|1\rangle \\
& X|1\rangle=\left[\begin{array}{ll}
0 & 1 \\
1 & 0
\end{array}\right]\left[\begin{array}{l}
0 \\
1
\end{array}\right]=\left[\begin{array}{l}
1 \\
0
\end{array}\right]=|0\rangle
\end{aligned}
$$

The Hadamard $(\mathrm{H})$ gate turns $|0\rangle$ and $|1\rangle$ into a equally weighted superposition states. When we apply $H$ to $|0\rangle$ and $|1\rangle$ we obtain the following superpositions:

$$
\begin{aligned}
& H|0\rangle=\frac{1}{\sqrt{2}}\left[\begin{array}{rr}
1 & 1 \\
1 & -1
\end{array}\right]\left[\begin{array}{l}
1 \\
0
\end{array}\right]=\frac{1}{\sqrt{2}}\left[\begin{array}{r}
1 \\
1
\end{array}\right] \\
& H|1\rangle=\frac{1}{\sqrt{2}}\left[\begin{array}{rr}
1 & 1 \\
1 & -1
\end{array}\right]\left[\begin{array}{l}
0 \\
1
\end{array}\right]=\frac{1}{\sqrt{2}}\left[\begin{array}{r}
1 \\
-1
\end{array}\right]
\end{aligned}
$$

The superposition principle allows us to carry out a computation on a qubit, in a superposition state, which indirectly affects all basis states that contribute to the superposition.

When it is necessary to work with two or more qubits, the concept of quantum register emerges. For two qubits, the state of the quantum system is defined by the tensor product of the single qubits as shown below:

$$
|00\rangle=\left[\begin{array}{l}
1 \\
0
\end{array}\right] \otimes\left[\begin{array}{l}
1 \\
0
\end{array}\right]=\left[\begin{array}{c}
1 \times\left[\begin{array}{l}
1 \\
0
\end{array}\right] \\
0 \times\left[\begin{array}{l}
1 \\
0
\end{array}\right]
\end{array}\right]=\left[\begin{array}{l}
1 \\
0 \\
0 \\
0
\end{array}\right]
$$

What means that a 2-qubit system or quantum register resides in a four dimensional Hilbert space whose basis are:

$$
\begin{aligned}
& |00\rangle=\left[\begin{array}{l}
1 \\
0 \\
0 \\
0
\end{array}\right]|01\rangle=\left[\begin{array}{l}
0 \\
1 \\
0 \\
0
\end{array}\right] \\
& |10\rangle=\left[\begin{array}{l}
0 \\
0 \\
1 \\
0
\end{array}\right]|11\rangle=\left[\begin{array}{l}
0 \\
0 \\
0 \\
1
\end{array}\right]
\end{aligned}
$$

Within this four dimensional space an arbitrary separable superposition state $|\psi\rangle$ can be written as:

$$
|\psi\rangle=\frac{1}{\sqrt{2}}(|00\rangle+|10\rangle)
$$

A two qubit quantum system, in a superposition state, is said to be separable [3] if it can be decomposed and expressed as a tensor product of the individual basis state vectors. For example:

$$
|\psi\rangle=\frac{1}{\sqrt{2}}(|00\rangle+|10\rangle)=\frac{1}{\sqrt{2}}(|0\rangle+|1\rangle) \otimes|0\rangle
$$

However, a superposition state $|\psi\rangle$ of a two qubit quantum system can be in a non-separable or entangled state and when this occurs, we are unable to decompose the state and express it as a tensor product of the individual basis state vectors. For instance, the following state represents an entanglement.

$$
|\psi\rangle=\frac{1}{\sqrt{2}}(|01\rangle+|10\rangle)
$$

The CNOT gate is of central interest to quantum computing because it produces entanglement. As it is illustrated in Figure 1, the CNOT gate has two input qubits, the one on top is denoted as the control qubit and the other one is known as the target qubit. When the control qubit is in state $|0\rangle$, both qubits just move across the CNOT unaltered but if the control qubit is in state $|1\rangle$ the control qubit moves across CNOT as is and the target qubit is flipped.

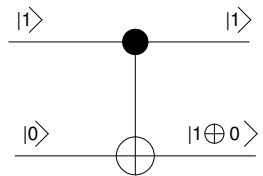

Figure 1. The CNOT gate .

In its matrix form the CNOT can be represented as a $4 \times 4$ matrix with a bandwidth of 3 .

$$
\text { CNOT }=\left[\begin{array}{llll}
1 & 0 & 0 & 0 \\
0 & 1 & 0 & 0 \\
0 & 0 & 0 & 1 \\
0 & 0 & 1 & 0
\end{array}\right]
$$

The evolution of a n-qubit quantum system in an initial superposition state can be described by a $2^{n} \times 2^{n}$ unitary matrix. However, this evolution can be described as well as a composition of single-qubit and controlled-not unitary operations which are equivalent to the $2^{n} \times 2^{n}$ unitary matrix. For instance,

$$
H^{\otimes 2}=(H \otimes H)=\frac{1}{2}\left[\begin{array}{cccc}
1 & 1 & 1 & 1 \\
1 & -1 & 1 & -1 \\
1 & -1 & -1 & -1 \\
1 & 1 & -1 & 1
\end{array}\right]
$$

can be decomposed into

$$
(H \otimes H)=\frac{1}{\sqrt{2}}\left[\begin{array}{rr}
1 & 1 \\
1 & -1
\end{array}\right] \otimes \frac{1}{\sqrt{2}}\left[\begin{array}{rr}
1 & 1 \\
1 & -1
\end{array}\right]
$$

The term quantum computation refers to the evolution of an isolated system of $n$ qubits by means of unitary operations which can be described by a quantum circuit[6]. A quantum circuit is a composition of quantum gates that implement an algorithm or is used to describe part of it. As shown in Figure 2, we could generalize that using one-qubit and two-qubit gates, a quantum circuit is arranged in a series of computational stages where each stage consists of the application of either one unitary operations acting on a single qubit, or two unitary operations acting in parallel on independent single qubits, or a two-qubit gate operating on a pair of qubits. Each stage represents a discrete time instant where unitary operations are applied and the progress of the algorithm can be seen as moving sequentially from one stage to another. 


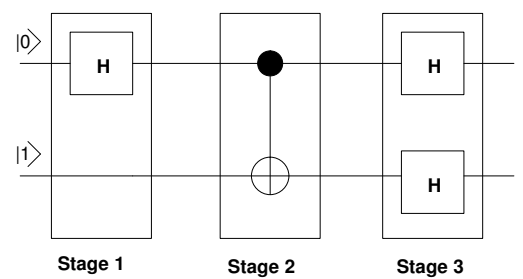

Figure 2. Schematic diagram of a quantum circuit.

\section{Systolic Quantum Circuits}

An alternative to solve the matrix vector product in parallel are systolic arrays. Kung and Leiserson[2] proposed a systolic array able to compute the banded matrix-vector product in $T(n)=w+2 n-1$ steps, where $w$ represents the number of Processing Elements (PE). Surprisingly, the same systolic array computes two matrix vector products simultaneously in $T(n)=w+2 n$ steps using perfect shuffling as a spatial data scheduling technique. Therefore, based in the mixed tensor product rule, $(H \otimes H)(|0\rangle \otimes|1\rangle)=H|0\rangle \otimes H|1\rangle$, it is possible create a separable superposition of two qubits in a single run. For instance, $H|0\rangle \otimes H|1\rangle$ will be carried out in 8 steps using the arrangement depicted in Figure 3. In this arrangement the components of $|0\rangle$ and $|1\rangle$ are merged, using perfect shuffling, into a single array that we will refer to as the carrier vector. The carrier vector contains the components of $|0\rangle$ stored in the odd locations and the components of $|1\rangle$ in the even ones. As illustrated in Figure 3, the circled components of $|1\rangle$ in the carrier vector are multiplied by the Hadamard matrix whose components are circled. The other Hadamard matrix is multiplied by $|0\rangle$.

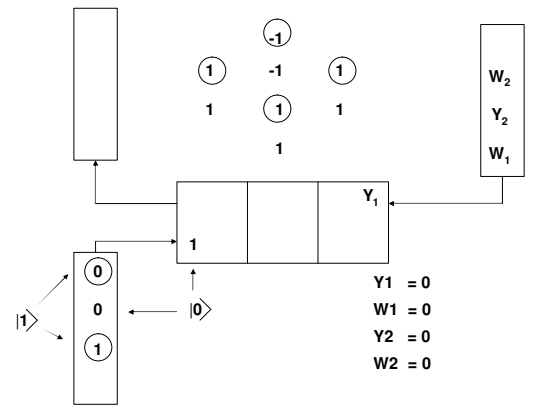

Figure 3. Executing two Hadamard gates in parallel.

The mapping of the CNOT gate onto the three PE systolic array is easily realizable. However, we will represent the CNOT transformation using a data adaptable gate to handle the two qubits in the carrier vector according to the following conventions: When the carrier vector inputs the CNOT gate the first and third elements of the carrier vector represent the control qubit and the second and third elements represent the target qubit. If the control qubit is $|1\rangle$ the CNOT gate will per- form $X \otimes I$ on the carrier vector, which means that an $I$ gate is applied on the first qubit of the carrier vector and a $X$ transformation on the second qubit. But when the control qubit is $|0\rangle$, CNOT performs $I \otimes I$. In the second step of the teleportation circuit, in section 4, we will show how the CNOT transformation is applied to $|10\rangle$. We use the first component of the control qubit as a selector of two data sets to implement either $X \otimes I$ or $I \otimes I$.

\section{Teleportation circuit}

The idea behind Quantum Teleportation [4] is to move quantum information around without destroying its quantum state. Imagine that Alice and Bob, share a classical communication channel and an entangled quantum state. For Alice to teleport a quantum state to Bob, she measures (and destroys) her share of the entangled state. This produces classical information that Alice sends to Bob. Depending upon the information received, Bob reconstructs Alice's quantum state by selectively applying a unitary operation to his shared entangled state. Figure 4 shows the basic quantum teleportation circuit.

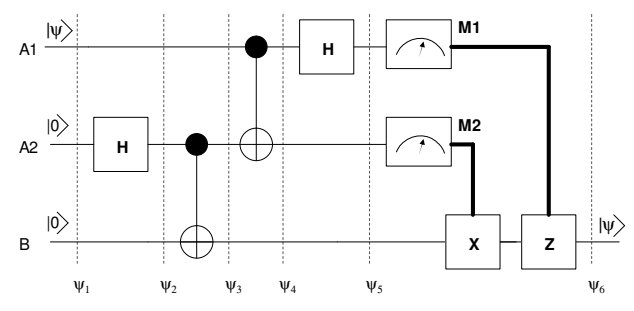

Figure 4. Quantum teleportation circuit.

The circuit performs unitary operations sequentially. They are primarily matrix-vector multiplication and we propose them to be carried out using systolic arrays. The input to each systolic array is a 1-dimensional vector denoted the carrier vector, hence we represent the qubits as 1-Dimensional vector as follows,

$$
|\psi\rangle=\alpha|0\rangle+\beta|1\rangle=\left[\begin{array}{c}
\alpha \\
D \\
\beta \\
D
\end{array}\right]|0\rangle=\left[\begin{array}{c}
1 \\
D \\
0 \\
D
\end{array}\right]|1\rangle=\left[\begin{array}{c}
0 \\
D \\
1 \\
D
\end{array}\right]
$$

where "D" stands for a don't care value. A 2-qubit state as a 1-dimensional vector is represented as one of the following carrier vectors:

$$
|00\rangle=\left[\begin{array}{l}
1 \\
1 \\
0 \\
0
\end{array}\right]|01\rangle=\left[\begin{array}{l}
1 \\
0 \\
0 \\
1
\end{array}\right]
$$




$$
|10\rangle=\left[\begin{array}{l}
0 \\
1 \\
1 \\
0
\end{array}\right]|11\rangle=\left[\begin{array}{l}
0 \\
0 \\
1 \\
1
\end{array}\right]
$$

The first and the third components of the carrier vector correspond to a qubit and the second and fourth components of the vector correspond to another qubit. The following description contains a step-by-step view of the teleportation circuit shown in Figure 4. The 2 lines on the top belong to Alice and we will refer to them as $A_{1}$ and $A_{2}$ and the line on the bottom belongs to Bob and we denote it as $B$.

1. $\left|\psi_{1}\right\rangle$ : The $A_{1}$ qubit is the unknown qubit state $|\psi\rangle=$ $\alpha|0\rangle+\beta|1\rangle$ to be teleported to Bob. The other 2 qubits, $A_{2}$ and $B$, simply have a $|0\rangle$ in the beginning. Thus the initial state is $|\psi\rangle \otimes|0\rangle \otimes|0\rangle$

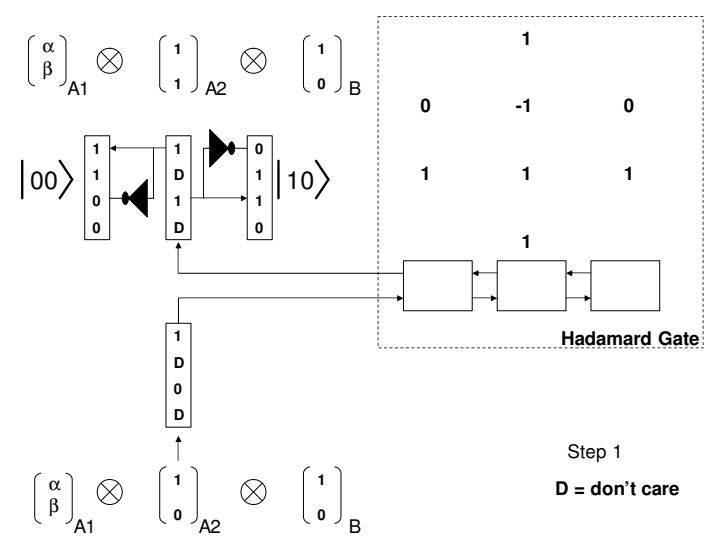

Figure 5. First step of the systolic teleportation design.

2. $\left|\psi_{2}\right\rangle$ : After the Hadamard operation is carried out on $A_{2}$ using the systolic array shown in Figure 5, the new 3-qubit system can be represented as $(\alpha|0\rangle+\beta|1\rangle) \otimes \frac{1}{\sqrt{2}}(|0\rangle+|1\rangle) \otimes$ $|0\rangle$. Note that the Hadamard gate is a 1-qubit gate and therefore we applied an identity transformation on the second qubit which is, in this case, a don't care qubit denoted as "D" in Figure 5. The 3-qubit system in state $\left|\psi_{2}\right\rangle$ can be rewritten as $(\alpha|0\rangle+\beta|1\rangle) \otimes \frac{1}{\sqrt{2}}(|00\rangle+|10\rangle)$

3. $\left|\psi_{3}\right\rangle$ : A CNOT operation is carried out on $A_{2}$ and $B$. This operation creates a shared entangled state between Alice and Bob. The $A_{2}$ qubit acts as a control qubit and $B$ qubit is the target qubit for the CNOT operation. It is worthwhile mentioning that the $A_{2}$ qubit is in a superposition state. Therefore, we separate the states into $|0\rangle$ and $|1\rangle$, as the control bits, to simulate the CNOT operation acting on $B$ qubit whose value is $|0\rangle$. The output observed at the systolic array is as follows, $C N O T|00\rangle=|00\rangle$ and $C N O T|10\rangle=>|11\rangle$, which represent the entangle state shared by Alice and Bob. The new 3-qubit system is now represented by $(\alpha|0\rangle+\beta|1\rangle) \otimes \frac{1}{\sqrt{2}}(|00\rangle+|11\rangle)$ as shown in Figure 6. Bob takes is entangled qubit $\frac{1}{\sqrt{2}}(|0\rangle+|1\rangle)$ and goes to Paris and Alice stays in New York.

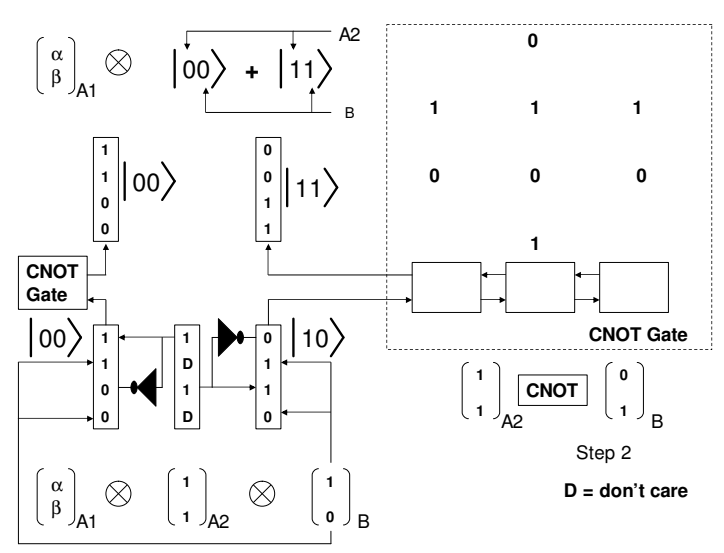

\section{Figure 6. Second step of the systolic teleporta- tion design .}

4. $\left|\psi_{4}\right\rangle$ : A CNOT operation is carried out on $A_{1}$ and $A_{2}$. This time $A_{1}$ is the control qubit and shared entangled qubit $A_{2}$ is the target qubit. To simulate the CNOT on $A_{1}$ and $A_{2}$ we rewrite the 3-qubit input as:

$$
\frac{1}{\sqrt{2}}[\alpha|\mathbf{0}\rangle(|\mathbf{0} 0\rangle+|\mathbf{1} 1\rangle)+\beta|\mathbf{1}\rangle(|\mathbf{0} 0\rangle+|\mathbf{1} 1\rangle)]
$$

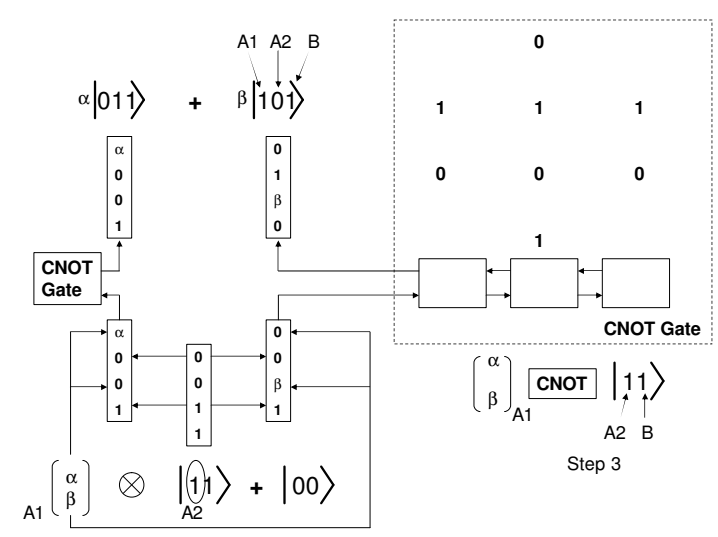

Figure 7. Third step of the systolic teleportation design .

where $A 1$ and $A 2$ are highlighted in bold type.

Then we carry out the CNOT operation on $\left(A_{1}, A_{2}\right)$, and thus the former state is transformed into:

$$
\left.\frac{1}{\sqrt{2}}[\alpha|\mathbf{0 0 0} 0\rangle+\alpha|\mathbf{0 1} 1\rangle)+\beta|\mathbf{1 1} 0\rangle+\beta|\mathbf{1 0 1} 1\rangle\right]
$$

Figure 7 shows the systolic CNOT operation applied to $\alpha|\mathbf{0 1 1}\rangle$ and $\beta|\mathbf{1 1} 1\rangle$ whose outcome is $\alpha|\mathbf{0 1 1}\rangle$ and $\beta|\mathbf{1 0 1}\rangle$. We show the $B$ qubit in the output in Figure 7 because that qubit is just passed on to the next stage preserving its state. 
5. $\left|\psi_{5}\right\rangle$ : Finally, a Hadamard operation is performed on the $A_{1}$ quibit to transform the $A 1$ state into a superposition. The former state is thus transformed into:

$$
\begin{aligned}
& \frac{1}{2}[\alpha(|\mathbf{0}\rangle+|\mathbf{1}\rangle)|00\rangle+\alpha(|\mathbf{0}\rangle+|\mathbf{1}\rangle)|11\rangle+\beta(|\mathbf{0}\rangle-|\mathbf{1}\rangle)|10\rangle+ \\
&\beta(|\mathbf{0}\rangle-|\mathbf{1}\rangle)|01\rangle]
\end{aligned}
$$

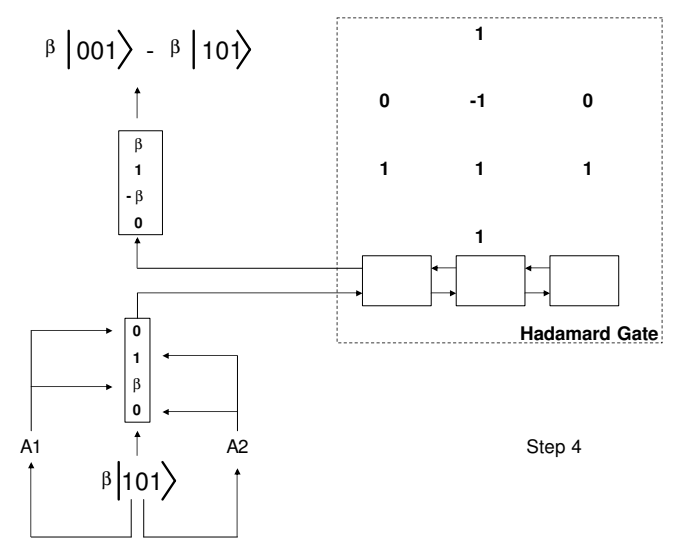

\section{Figure 8. Fourth step of the systolic teleporta- tion design .}

Figure 8 shows the Hadamard operation on the $A_{1}$ qubit in the state $\beta|\mathbf{1 0 1}\rangle$, whose resulting value is $\beta|\mathbf{0} 01\rangle-\beta|\mathbf{1 0 1}\rangle$. Actually, Alice ends up the with four carrier vectors similar to the one shown in Figure 8. Then, Alice's qubits $A_{1}$ and $A_{2}$ can be regrouped by factoring out the $B$ qubit to obtain the following expression:

$$
\begin{aligned}
& \left|0_{A 1} 0_{A 2}\right\rangle \frac{(\alpha|0\rangle+\beta|1\rangle)}{2}+\left|0_{A 1} 1_{A 2}\right\rangle \frac{(\alpha|1\rangle+\beta|0\rangle)}{2}+ \\
& \left|1_{A 1} 0_{A 2}\right\rangle \frac{(\alpha|0\rangle-\beta|1\rangle)}{2}+\left|1_{A 1} 1_{A 2}\right\rangle \frac{(\alpha|1\rangle-\beta|0\rangle)}{2}
\end{aligned}
$$

6. $\left|\psi_{6}\right\rangle$ : Alice's quantum bits are measured, as indicated in Figure 4 in the boxes $M 1$ and $M 2$. After the measurement the values of the two classical bits are sent to Bob using the shared classical communication channel.

7. $\left|\psi_{7}\right\rangle$ : Bob can receive one out of four different combination of 2 classical bits and a particular unitary transformation is applied to Bob's shared entangled state to recover the same quantum state, $|\psi\rangle$, that was on line $A_{1}$ prior to the beginning of the experiment. For instance, if Bob receives $|10\rangle$, then he applies $Z$ to his shared entangled state as shown below:

$$
\left[\begin{array}{rr}
1 & 0 \\
0 & -1
\end{array}\right]\left[\begin{array}{r}
\alpha \\
-\beta
\end{array}\right]=\left[\begin{array}{l}
\alpha \\
\beta
\end{array}\right]=|\psi\rangle
$$

In the other three cases when $|00\rangle$ is received, $I$ is applied, when $|01\rangle$ is received, $X$ is applied, and when $|11\rangle$ is received, Bob applies $X$ and then $Z$.

\section{Conclusions}

We have presented a new technique suitable to describe and simulate quantum circuits using systolic arrays as the basic building blocks. We have shown how the mapping of two simultaneous matrix-vector products onto a three PE systolic array, allows us a fast realization of the CNOT gate and the superposition of two independent qubits in parallel. Our preliminary results shows that the teleporting of a single qubit takes 32 steps using four systolic arrays. However, a HadamardCNOT gate can be realized to unify the last two stages into one reducing thus the computation time in $25 \%$. Other improvement that can be done is the bypassing of the CNOT operations where the control qubit is zero and this way the whole teleportation circuit can be executed with only two systolic arrays. We are currently interested in the efficient implementation of QCA simulators using our approach and the mapping of other quantum algorithms onto systolic arrays.

\section{References}

[1] N.D. Mermin, From Cbits to Qbits: Teaching Computer Scientists Quantum mechanics, American Journal of Physics Vol. 71, 2003, pp. 23-30.

[2] H. T. Kung and C. E. Leiserson, Systolic Arrays, In I. S. Duff and G. W. Stewart (Eds.) Sparse Matrix Proceeding, SIAM, 1978, pp. 256-282.

[3] G. Benenti, G. Casat, and G. Strini Principles of quantum computation and information, World Scientific Publishing Co. Pte. Ltd. 2004.

[4] Gilles Brassard, Samuel L. Braunstein, and Richard Cleve Teleportation as a quantum computation, Physica D, Volume 120, 1998, pp. 43-47.

[5] Peter W. Shor, Polynomial-Time Algorithms for Prime Factorization and Discrete Logarithms on a Quantum Computer,SIAM Journal on Computing Vol. 26, No. 5, 1997, pp. 1484-1509.

[6] M.A Nielsen and I.L. Chuang, Quantum Computation and Quantum Information, Cambridge Univ. Press, 2000

[7] M. Udrescu, L. Prodan and M. Vladutiu, Using DHLs for Describing Quantum Circuits: A Framework for Efficient Quantum Algorithm Simulation”, CF’04, Italy, 2004, pp. 96-110.

[8] A. U. Khalid, Z. Zilic and K. Radecka, FPGA Emulation of Quantum Circuits, IEEE International Conference on Computer Design (ICCD), 2004, pp. 310-315.

[9] G. F. Viamontes, M. Rajagopalan, I. L. Markov and J. P. Hayes, Gate Level Simulation of Quantum Circuits, ASPDAC, Japan, January 2003, pp. 295-301.

[10] David P. DiVincenzo, Quantum Gates and Circuits, Proc. R. Soc. London A: Mathematical, Physical and Engineering Sciences, Vol. 454, 1998, pp. 261-276.

[11] Bernhard Omer, A Procedural Formalism for Quantum Computing, Master thesis, Department of Theoretical Physics, Technical University of Vienna, 1998. 